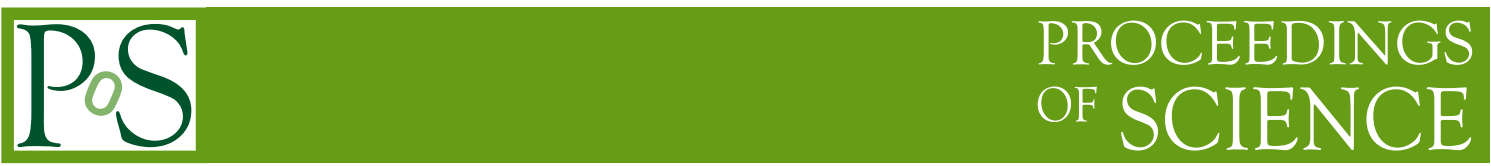

\title{
Did Dark Matter Kill the Dinosaurs?
}

\author{
Michael Sarkis* \\ School of Physics, University of the Witwatersrand, Private Bag 3, WITS-2050, Johannesburg, \\ South Africa \\ E-mail: michael.sarkisestudents.wits.ac.za
}

\section{Geoff Beck}

School of Physics, University of the Witwatersrand, Private Bag 3, WITS-2050, Johannesburg, South Africa

E-mail: geoffery.beckewits.ac.za

\begin{abstract}
Potential links between astrophysical sources, such as Gamma-Ray Bursts (GRBs) and Supernovae (SN), and mass extinction events on Earth are of interest in the historical trajectory of life on our planet. There are strong arguments to suggest that these astrophysical sources can have several destructive effects, including depletion of atmospheric ozone and an increase in the radiation dose received by living organisms. Recent atmospheric simulations have shown that the fluence from a typical GRB at a distance of $\sim 2 \mathrm{kpc}$ has the potential to deplete up to $38 \%$ of global atmospheric ozone, with as much as $74 \%$ localised depletion. In this work, it is hypothesised that the annihilation of Weakly Interacting Massive Particles (WIMPs) in the core of a clump of dark matter that passes through our solar system could be the source of a gamma-ray fluence at the surface of the Earth that is of a similar magnitude to nearby GRBs. It was found that extremely dense clumps of dark matter, so-called Ultra-compact Minihalos, can produce a much larger fluence, potentially enhancing this effect. The implications of this would be a large flux of solar UV-B radiation able to penetrate the atmosphere, leading to widespread cancer formation and DNA damage, as well as significant changes to climate. These effects are compatible with extinction patterns found from the Late Ordovician extinction event that occurred $\sim 443$ My ago.
\end{abstract}

High Energy Astrophysics in Southern Africa - HEASA2018

1-3 August, 2018

Parys, Free State, South Africa

* Speaker. 


\section{Introduction}

It has recently been suggested that a nearby GRB acted as a trigger for the mass extinction event in the Late-Ordovician era roughly $443 \mathrm{My}$ ago [1,2]. If the Earth were in the path of the radiation jets of a GRB, high-energy photons would be deposited into the Earth's atmosphere that could significantly affect atmospheric chemistry. Of the resulting changes, a reduction in the amount of ozone $\left(\mathrm{O}_{3}\right)$ in the stratosphere is arguably the most detrimental to living organisms, as ozone molecules absorb almost all of the solar radiation in the UV-B band and any loss of ozone would lead to an increased flux of UV-B reaching the surface. This radiation is particularly harmful to living organisms, as it is absorbed by DNA molecules and can cause the onset of genetic mutations and cancers [3]. This could influence the food chain and biodiversity on a large scale if primary food producers like phytoplankton are affected, and it is argued in [2] that the extinction patterns evident in the Late-Ordovician era match the effects of an astrophysical ionising event like a GRB.

When investigating these effects, simulations of the atmosphere's response to GRBs of typical size and distance from the Earth were carried out by [1] using the Goddard Space Flight Center 2D atmospheric model. The results of these simulations showed significant amounts of ozone depletion, with the global average amount of ozone dropping up to $38 \%$. From these results an estimate of the amount of biological damage to DNA from increased levels of UV-B was found to be up to 16 times the annual global average in some locations - again a highly significant result when considering primary food producers. Several studies that consider SN (rather than GRBs) as sources of ionising radiation have also been performed $[4,5,6,7]$ and show similar levels of ozone depletion.

Recent work has brought attention to the possibility of Dark Matter (DM) affecting the Earth, most notably through gravitational perturbations when the solar system passes through a 'dark disk' of DM in the galactic plane [8,9] and heat generation by capture of DM particles from the galctic halo $[10,11]$. This work introduces the hypothesis that clumps of DM in the galaxy can, through annihilation and decay of WIMPs, act as sources of ionising radiation able to produce the atmospheric effects mentioned above. Evidence for these clumps - areas of high DM density compared to the surrounding galactic halo - is provided by several numerical simulations, summarised in [12]. While the exact distribution of clumps in the Milky Way is not yet known with much certainty, we find that clumps that pass close to or even within the solar system produce a total fluence of gamma rays comparable to that of the GRB considered in [1]. This is true for a range of possible WIMP masses and annihilation/decay channels, with gamma-ray fluences increasing for more massive clumps.

The structure of this paper will be as follows: in Sec. 2 we outline the most relevant aspects of gamma-ray interactions with the Earth's atmosphere and in Sec. 3 we present the mechanism for production of gamma rays from a general DM distribution. The specific type of DM halo used is then presented in Sec. 4. The results of this work and a discussion thereof are shown in Sec. 5 and Sec. 6 respectively, and concluding remarks are given in Sec. 7 .

\section{Effects of a gamma-ray flux on the Earth's Atmosphere}

An important consequence of a gamma-ray flux on the Earth's atmosphere is the production of nitrogen oxides, especially of $\mathrm{NO}$ and $\mathrm{NO}_{2}$. Ionising radiation produces free nitrogen atoms that, 
through the reaction

$$
\mathrm{N}+\mathrm{O}_{2} \longrightarrow \mathrm{NO}+\mathrm{O}
$$

form $\mathrm{NO}$ molecules. The interaction between these $\mathrm{NO}$ molecules and other nitrogen oxides is dynamic and complex, depending on solar irradiance levels and the relative abundances of each compound (see for example $[13,14]$ ), but the formation of NO has a notable effect on ozone abundance.

The number of $\mathrm{O}_{3}$ molecules present in a thin layer in the Earth's stratosphere fluctuates naturally as they interact with other chemical species in the atmosphere. The reactions that are pertinent to this research include those that reduce the total number of ozone molecules, which are often referred to as catalytic cycles [15]. The cyclic nature of these reactions can be seen by the following example using $\mathrm{NO}$ - noting that cycles involving other species like $\mathrm{OH}$ and $\mathrm{Cl}$ proceed in a similar way:

$$
\begin{aligned}
& \mathrm{NO}+\mathrm{O}_{3} \longrightarrow \mathrm{NO}_{2}+\mathrm{O}_{2} \\
& \mathrm{NO}_{2}+\mathrm{O} \longrightarrow \mathrm{NO}+\mathrm{O}_{2}
\end{aligned}
$$

Here the NO molecule which is responsible for dissociating the $\mathrm{O}_{3}$ is preserved throughout the cycle, while the $\mathrm{O}_{3}$ molecule is lost. It is possible for these reactions to repeat themselves, even up to several hundreds of times [6], before the NO molecule interacts with a different atmospheric species. In this way, ozone can be catalytically depleted even with a relatively low abundance of NO.

\section{Gamma rays from DM}

Gamma-ray photons are one of several kinematically possible WIMP annihilation/decay products. The calculation of the prompt flux of gamma rays from a clump of DM, as summarised in [16], is governed by the equations

$$
\frac{d \Phi}{d E}= \begin{cases}\frac{\langle\sigma v\rangle}{8 \pi m_{\chi}^{2}} J \sum_{f} \frac{d N_{x}^{f}}{d E} B_{f}, & \text { (annihilation) } \\ \frac{\Gamma}{4 \pi m_{\chi}} D \sum_{f} \frac{d N_{x}^{f}}{d E} B_{f}, & \text { (decay). }\end{cases}
$$

The quantities $\langle\sigma v\rangle$ and $\Gamma$ represent the WIMP's velocity-averaged pair annihilation cross section and decay rate, respectively, while $m_{\chi}$ is the particle mass. In this work the thermal relic cross section value of $\langle\sigma v\rangle \sim 3.0 \times 10^{-26} \mathrm{~cm}^{3} \mathrm{~s}^{-1}$ and decay rate of $\Gamma \sim 4.0 \times 10^{-26} \mathrm{~s}^{-1}$ are used with a range of WIMP masses [17].

The equations in Eqn. 3.1 each have contributions from both the particle nature of WIMPs as well as the overall halo structure. The differential energy spectrum of photons produced by a single annihilation/decay, through a channel labelled by $f$ and with a branching ratio of $B_{f}$, is given by the factor $\frac{d N_{x}^{f}}{d E}$ with the sum running over all possible annihilation/decay channels. During the calculations one channel is selected at a time, and the spectra are obtained from the numerical results 
of [16]. The contribution to the flux from the halo structure is represented by the quantities labelled $J$ and $D$. These are usually expressed as the integral of the DM density over the line-of-sight (los) to the halo, with

$$
J=\int_{l o s} \rho^{2}(\mathbf{r}) d l
$$

and

$$
D=\int_{l o s} \rho(\mathbf{r}) d l
$$

\section{Ultracompact Dark Matter Minihalos}

As can be seen in Eqn. 3.2, the gamma-ray flux from a DM halo depends strongly on the density profile, especially in the case of annihilation. With this in mind, it is worth studying any potentially nearby halos that have highly dense cores. The so-called ultra-compact minihalo (UCMH) profile, first proposed as a hypothetical dark MAssive Compact Halo Object (MACHO) by Ricotti and Gould in 2009 [18], possesses a characteristically steep radial dependence given by

$$
\rho(r, z)=\frac{3 f_{\chi}}{16 \pi} \frac{M_{\mathrm{UCMH}}(z)}{R_{\mathrm{UCMH}}(z)^{\frac{3}{4}}} \frac{1}{r^{\frac{9}{4}}},
$$

making it a suitable choice for this work. Here $f_{\chi}$ gives the fraction of total matter in the universe that is dark, while $M_{\mathrm{UCMH}}$ and $R_{\mathrm{UCMH}}$ are the virial mass and radius of the halo at a redshift $z$. The density at the centre of the halo is suppressed by DM annihilations however, with a maximum estimated value [19] of

$$
\rho_{\max } \simeq \frac{m_{\chi}}{\langle\sigma v\rangle\left(t_{0}-t_{c}\right)},
$$

where $t_{0}$ is the age of the universe and $t_{c}$ is the collapse time of the halo. As in [20], the density of the halo at any radius is taken as the maximum value between the results of Eqn. 4.1 and Eqn. 4.2. It is estimated that accretion onto the UCMH ends at a redshift $z \sim 10$, at which point the halo stops growing and has a virial radius of

$$
R_{\mathrm{UCMH}}(z)=0.019\left(\frac{1000}{z+1}\right)\left(\frac{M_{\mathrm{UCMH}}(z)}{M_{\odot}}\right)^{\frac{1}{3}} \mathrm{pc} .
$$

This work considers halos with masses in the range $1 M_{\odot}-100 M_{\odot}$, which are large compared to the size of the solar system and appear as extended sources for all distances considered. A standard halo velocity dispersion of $300 \mathrm{~km} / \mathrm{s}$ (following the bulk galactic DM) is used.

\section{Results}

The gamma-ray flux calculated from Eqn. 3.1 for a single halo was integrated over a timespan of 10 days and over the energy range given by the spectra $d N_{\chi}^{f} / d E$ from [16]. The results are set to display the minimum distance to the halo for a certain threshold of gamma-ray fluence to be received by the Earth, for a range of UCMH masses. The fluence values of 10,100 and $1000 \mathrm{~kJ} / \mathrm{m}^{2}$ correspond to those considered for the case of a nearby GRB and that show significant levels of 

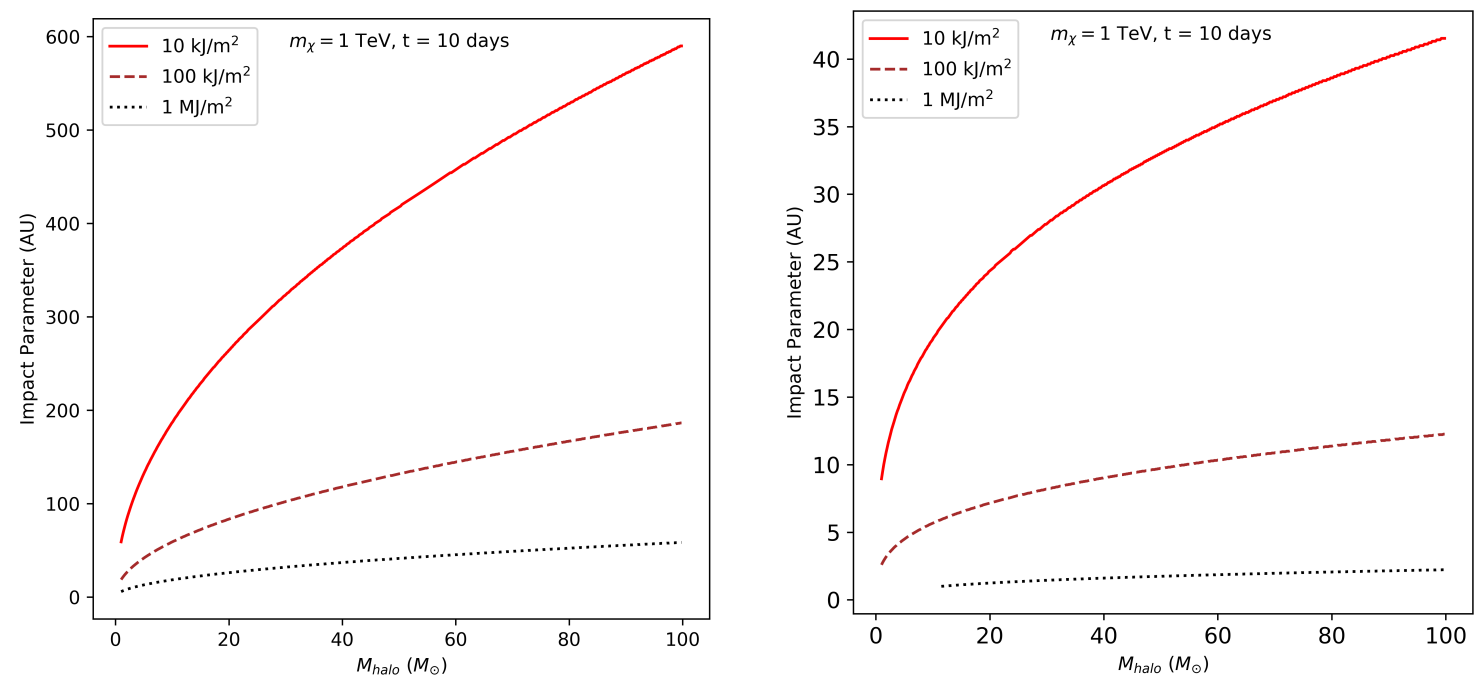

Figure 1: Computed minimum impact parameter to an UCMH that yields a gamma-ray fluence of the indicated threshold through WIMP annihilation (left) and decay (right). Results are obtained using the thermal relic annihilation cross section and decay rate through the light quark channel.

ozone depletion in the atmospheric simulations carried out by [1]. Results for a WIMP mass of 1 $\mathrm{TeV}$, annihilating into gamma rays through light quarks, are shown in Figure 1.

The results displayed here are obtained using a set of realistic WIMP and halo parameters. These values are subject to change as they are better constrained in the future, and different values to the ones used here can, in general, lead to different fluence values. In particular, the distance to the halo should scale with the annihilation cross section and decay rate by at least as $\langle\sigma v\rangle^{-\frac{1}{2}}$ for annihilation and $\Gamma^{-\frac{1}{2}}$ for decay. Different WIMP masses lead to relatively small changes in the results, with the minimum distance to the halo only changing by a factor of $\sim 3$ over a range of WIMP masses between $10 \mathrm{GeV}$ and $10 \mathrm{TeV}$ (this is explained by halos in the mass range considered here having enough mass for the density in the central region to reach the maximum value given in Eqn. 4.2 which, containing a factor of $m_{\chi}$, cancels the dependence on the WIMP mass in Eqn. 3.1). Variations in the time of collapse for the halo, between redshifts of $z=200$ and $z=3000$, result in changes to the annihilation flux of less than $1 \%$.

\section{Discussion}

While these results show a possibility for nearby DM halos to affect the Earth's atmosphere, the magnitude of this effect can only be roughly compared to results of previous studies focused on supernovae and GRBs. Even for equal gamma-ray fluences, there are notable differences in the way the energy is deposited into the atmosphere. Firstly, the impact area on the atmosphere from highly directed sources like GRBs would be more concentrated than that of a nearby DM halo, and it seems likely that this would lead to variations in the ozone depletion at latitudes near the impact. Secondly, the different time intervals of impacts from different sources (receiving the total energy from slow moving DM halos would take longer than a typical GRB) could lead to changes in the effective recovery time of ozone levels. However, if the impact time is much shorter than the timescale for 
ozone recovery this difference could be negligible. These uncertainties should be addressed with full atmospheric simulations of the type mentioned above.

The type of DM halo used in this work was chosen for its steep density profile and comparitevely high gamma-ray yield. The non-observation to date of a halo of this type leaves some uncertainty in the exact form of the profile, and recent simulations [21] suggest the density of the inner regions of the halo might follow a shallower profile. This does not change the overall mechanism we have considered, but preliminary tests with low-mass halos indicate that the flux output is roughly two orders of magnitude weaker than UCMHs. The distribution of UCMHs throughout the galaxy, and hence the rate of encounters with the Earth, is also currently uncertain as the fraction of galactic DM contained inside these halos is not well known [20], and a study of this should be the subject of future work.

\section{Conclusion}

The results of this work indicate a strong possibility that gamma rays produced by annihilating WIMPs inside galactic DM clumps can reduce the amount of ozone in the Earth's atmosphere. This effect is enhanced for clumps that pass nearby the solar system as well as highly dense clumps, such as UCMHs. While the results can be roughly compared to previous studies that investigate a similar mechanism involving GRBs and SN, full atmospheric simulations would provide a more accurate set of climactic and biological consequences.

Additionally, there is currently a high level of uncertainty in the presence and location of these clumps. Any estimates for the rate at which they pass near enough to the solar system for globally significant effects would be too inaccurate for a definite answer to the question posed in the title. However, with current evidence it seems at least plausible that encounters between DM clumps and the Earth have occurred since the formation of the solar system, potentially affecting the development of life on Earth.

\section{Acknowledgments}

This work is based on the research that was supported by the South African Research Chairs Initiative of the Department of Science and Technology and National Research Foundation of South Africa (Grant No 77948 \& Bursary No 112332).

\section{References}

[1] Thomas B, et al. 2005 ApJ 634 509-533

[2] Melott A and Thomas B 2009 Paleobiology 35.3 311-320

[3] Setlow R 1974 Proc. Nat. Acad. Sci. USA 71.9 3363-3366

[4] Ruderman M 1974 Science 184 1079-1081

[5] Ellis J and Schramm D 1995 Proc. Nat. Acad. Sci. USA 92 235-238

[6] Gehrels N, et al. 2003 ApJ 585 1169-1176

[7] Melott A, et al. $2017 \mathrm{ApJ} 8 \mathbf{8 4 0}$ 105-114 
[8] Randall L and Reece M 2014 Phys. Rev. Lett. 112161301

[9] Kramer E and Rowan M 2017 eprint arXiv:1610.04239

[10] Abbas S and Abbas A 1998 Astroparticle Physics 8 317-320

[11] Rampino M 2015 MNRAS 448 1816-1820

[12] Kuhlen M, Vogelsberger M and Angulo R 2012 Dark Universe 1 50-93

[13] Brasseur G and Nicolet M 1973 Planetary and Space Science 21.6 939-961

[14] Vitt F and Jackman C 1996 J. Geophys. Res. 101 D3 6729-6739

[15] Madronich S 1993 Environmental UV Photobiology Plenum Press, NY ch1 p1-39

[16] Cirelli M, et al. 2011 JCAP 03051

[17] Steigman G, Dasgupta B, and Beacom J 2012 Phys. Rev. D 86023506

[18] Ricotti, M and Gould, A 2009 ApJ 707 979-987

[19] Ullio P, et al. 2002 Phys. Rev. D 66123502

[20] Bringmann T, Scott P and Akrami Y 2013 Phys. Rev. D 85125027

[21] Delos M, et al. 2018 Phys. Rev. D 98063527 